\title{
Relation between the severity of early malnutrition and the effects of later frontal cortical lesions in rats
}

\author{
ABDELAZIZ BOUZRARA, MARIO SILVA, DANIEL WAKSMAN, \\ STANLEY FINGER, and C. ROBERT ALMLI \\ Washington University, St. Louis, Missouri
}

\begin{abstract}
Rats with an early history of severe malnutrition received frontal cortical (FC) lesions or control operations at 90 days of age. These severely malnourished groups were compared with each other and with moderately malnourished and well-fed groups (FC and control) for acquisition and three reversals of a tactile discrimination (rough-smooth). Main effects of lesion and nutrition were found for acquisition and reversals. The groups with malnutrition plus frontal lesions displayed the poorest performance. Although the severely and moderately malnourished groups did not differ in acquisition of the discrimination, the severely malnourished group made more reversal errors. The severely malnourished rats also showed greater brain growth retardation (size and weight) than the moderately malnourished rats. These results indicate that the effects of early dietary history and the reaction to a later, acute brain injury may be additive under certain circumstances.
\end{abstract}

It is becoming increasingly apparent that nutritional insults to the developing nervous system can have long-term consequences. Some of the deficits associated with early undernutrition in animals include a reduction of physical growth (Eichenwald \& Fry, 1969; Resnick, Morgane, Hasson, \& Miller, 1982), decreased myelination (Davidson \& Dobbing, 1966), and smaller brains with reduced cell division (Winick, 1976).

One method for studying the effects of early undernutrition under controlled conditions involves giving isocaloric diets differing in protein content to pregnant and/or lactating dams and testing for differences among the offspring, which may or may not be maintained on the same diet as the dam during the postnatal period. Resnick and his co-workers (Miller \& Resnick, 1980; Resnick et al., 1979; Stern, Resnick, \& Morgane, 1976) found that when compared with well-fed offspring (dams fed a $25 \%$ casein diet), rat offspring of dams fed a lowprotein diet (8\% casein) displayed central and peripheral metabolic changes, including increased levels of tryptophan, serotonin, norepinephrine, and fatty acids, and that the malnourished rats showed poorer scores on a memory

Support for this investigation came from Grants BRSG-SO7-RR0705417 and -19 (Biomedical Research Support Grant Program) to S. Finger and Grant HD-06364 (NICHD) to C. Robert Almli (Worcester Foundation for Experimental Biology, Shrewsbury, MA). A Bouzrara was supported by the Scientific Mission of Tunisia; M. Silva was supported by NIA Training Grant AG 00030-07. S. Finger also has an appointment in the Neural Sciences Program at Washington University, and C. Robert Almli also has appointments with the Programs of Occupational Therapy and Neural Sciences, and in the Departments of Anatomy and Neurobiology and Preventive Medicine, Washington University School of Medicine, St. Louis, MO 63110. task. In addition, the malnourished rats showed decreased synaptic spine densities, reduced dendritic lengths (West \& Kemper, 1976), and altered olfactory bulb electrical activity development (Almli, Henault, Velozo, \& Morgane, 1984). Many of these abnormalities persist even if nutritional rehabilitation is started at birth (e.g., Resnick et al., 1979).

Work now has been initiated on an animal model for low-birth-weight human infants in which rat dams are maintained on a very-low-protein diet $(6 \%$ casein) throughout the gestational period (Almli \& Montoya, 1982; Almli \& Yang, 1983; Resnick \& Morgane, 1983). This very-low-protein diet results in offspring displaying significantly lower birth weights than control rats, whereas the low-protein diet discussed above produces offspring that have normal birth weights. The low-birth-weight offspring have been found to be more severely affected than the normal-birth-weight malnourished offspring on measures of physical growth, brain weight, and neurochemistry (Lundberg, Almli, Morgane, Motamedi, \& Cattegno, 1983; Resnick \& Morgane, 1983). In addition, the low-birth-weight offspring have been shown to display persistent developmental delays in sensorimotor performance (Almli \& Montoya, 1982), even when cross-fostered to well-fed dams at birth (Almli \& Yang, 1983).

Recently, we have become interested in the relation between early nutritional history and recovery from brain damage sustained later in life, the hypothesis being that early dietary history may be one factor influencing the variability in performance typically seen after "comparable" lesions in clinical populations (Finger \& Stein, 1982). In one test of this hypothesis, rat offspring of dams that were fed inadequate amounts of a normal rat diet showed 
poorer retention on a brightness-discrimination problem after lesions of the posterior cortex, even though the two nutritional groups performed equivalently prior to brain damage (Mangold, Bell, Gruenthal, \& Finger, 1981).

In a recent follow-up of the Mangold et al. (1981) study, low protein ( $8 \%$ casein) and normal protein diets were fed to dams 5 weeks prior to conception and throughout lactation, with some of the offspring receiving lesions of the frontal association cortex at maturity (Silva, Bouzrara, Finger, \& Almli, 1984). Main effects of undernutrition and the acute brain lesions were found when the animals were tested for acquisition of a simple tactile discrimination, with the "undernourished plus frontal cortex" group performing more poorly than any other group. There were no differences between groups, however, on a series of reversals of this task. Severely malnourished (low-birthweight, $6 \%$-casein-diet) rats were to have been included in this experiment. Unfortunately, these animals showed an unexpectedly high mortality rate during and immediately after the surgical operations that were performed when they were mature. Thus, the severely malnourished groups had to be dropped because of inadequate numbers.

We have now been able to study severely malnourished (low-birth-weight, $6 \%$ casein diet) rats under procedures nearly identical to those of Silva et al. (1984). Because these offspring were extremely small and immature at 3 weeks after birth, we delayed weaning them from their dams until 40 days postnatal in an attempt to reduce the high mortality rate of the previous study. This procedural change was successful in reducing (but not eliminating) the high mortality rate, and the behavioral and anatomical effects associated with severe malnutrition will be described. Further, these severely malnourished animals will be compared to the moderately malnourished $(8 \%$ casein) and well-fed rats tested under identical conditions by Silva et al. (1984), in order to achieve a better understanding of the relationship between the severity of early malnutrition and learning, brain growth, and the response to an acute cortical lesion sustained later in life.

\section{METHODS}

The procedures employed in this study were basically identical to those of Silva et al. (1984), except for the conditions of the dietary treatment. The animals studied were 20 male and 17 female albino rat (Charles River, "CD" strain) offspring from 11 litters. The offspring were obtained from dams exposed to a very low protein diet $(6 \%$ casein, Teklad Mills, Madison, WI), beginning 5 weeks before breeding. Pups from different litters were randomly crossfostered on the day of birth, and the litters were culled to 8 pups each. The pups were housed with their dams and maintained on the $6 \%$ casein diet until 40 days of age. At this age, the dams were removed and the offspring were housed by threes according to sex and given free access to Purina Rat Chow (23\% casein). These rats will henceforth be referred to as the severely malnourished (SM) animals. The normal-birth-weight, $8 \%$-casein-diet rats studied previously (Silva et al., 1984) will be termed the moderately malnourished (MM) rats, and the $25 \%$-casein-diet rats from the same study will be referred to as well-fed (WF) rats for purposes of comparison.
When they were 90 days of age, 14 rats ( 7 males and 7 females) were randomly assigned to receive one-stage, bilateral frontal cortex lesions (aspiration); 13 rats ( 9 males and 4 females) received control operations (surgery without skull damage or brain lesions). Rats of both the frontal cortex lesion (FC) and control operation (CO) groups were enucleated during these operations. The rats were then given a 10-day recovery period with continued free access to the standard laboratory chow and water. Among the 27 subjects, 6 with lesions ( 4 males and 2 females) and 9 with sham operations ( 7 males and 2 females) were tested. The remaining animals either died under the anesthetic (sodium pentobarbital; $40 \mathrm{mg} / \mathrm{kg}$ ) or during the postoperative recovery period. Although substantial, the mortality rate was lower than that encountered previously when trying to perform surgery on rats from the $6 \%$ casein dietary treatment.

After the recovery period, the two groups of rats began roughsmooth discrimination testing in a $\mathrm{T}$-maze. The enclosed singleunit T-maze had a startbox $(36 \times 23 \times 14 \mathrm{~cm})$ and two alleys $(64 \times$ $22 \times 14 \mathrm{~cm})$. On the floor of one alley was a smooth aluminum plate, and on the other was a "rough" aluminum plate (milled with spiral cuts of $0.5 \mathrm{~mm}$ made with a flying cutter (Finger \& Frommer, 1968). A dish of food (ground Purina Rat Chow mixed with water) served as the reward. The apparatus was housed in a soundattenuated room, and the plates were cleaned with Lysol solution and rinsed with water after trials.

The rats were food-deprived (24-h food-deprivation schedule) beginning one day before rough-smooth discrimination testing, with half of the rats initially assigned to the smooth tactile plate and the remainder assigned to the rough plate. The rats were rewarded with $30 \mathrm{sec}$ of access to food when the correct response was made. The location of the floor plates and the order in which the rats were run were randomly determined. Ten minutes of free access to wet chow completed each day of testing.

The rats received 5 trials/day, and the criterion for learning the tactile discrimination was a perfect score on 2 successive days. Upon reaching criterion, each rat was required to change plate preference in order to receive food, and this reversal was followed by two more reversals using the same criterion for learning. A period of 40 days without reaching criterion was used to terminate testing if criterion could not be met on a problem. These rats received a conservative maximum score of 42 days to criterion for statistical treatment of their data.

Analyses of variance were used to compare the scores of the present severely malnourished (SM) groups with those of the moderately malnourished (MM) and well-fed (WF) groups, since this was the original intent of the study by Silva et al. (1984).

Upon completion of testing, the rats (approximately 200 days of age) were killed by anesthetic overdose and their brains were processed for histological study. The olfactory bulbs anterior to the frontal cortex were removed. A second coronal cut was made between the occipital cortex and the cerebellum, and a third coronal cut was made just behind the cerebellum at the level of the medulla. The width and length (in centimeters) of the cerebral cortex were measured at their largest expanse, and the cerebellar and cerebral blocks were weighed individually. Drawings were made of the lesions, after which frozen sections were cut at $40 \mu$ on a microtome. Every seventh section was saved and stained with cresyl-violet for light microscopic examination.

\section{RESULTS}

\section{Body Weights}

The male rats in the SM condition had a mean body weight of $271.8 \mathrm{~g}$ and the female rats weighed $185.9 \mathrm{~g}$ at the time of surgery ( 90 days of age). Analyses of variance and subsequent Tukey tests showed that bociy weights of the SM rats were significantly lower than those of the 
$\mathrm{MM}$ rats $($ males $=332.3 \mathrm{~g}$, females $=221.3 \mathrm{~g} ; \mathrm{p}<.01$ and $\mathrm{p}<.05$, respectively). Body weights also differed significantly from the WF animals (males $=400.9 \mathrm{~g}$, females $=216.6 \mathrm{~g} ; \mathrm{ps}<.01$ ).

\section{Behavioral Measures}

The testing scores of the males and females were combined, and the animals first rewarded on the rough sur-

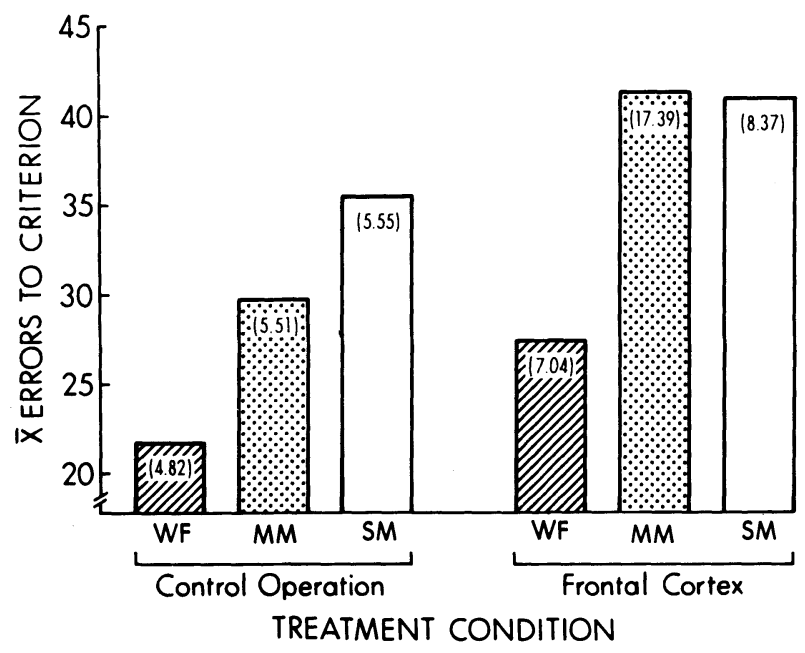

Figure 1. Performance scores (mean number of errors to criterion) of the six groups of rats in original learning of the tactile discrimination. The number in brackets at the top of each bar is the standard deviation.

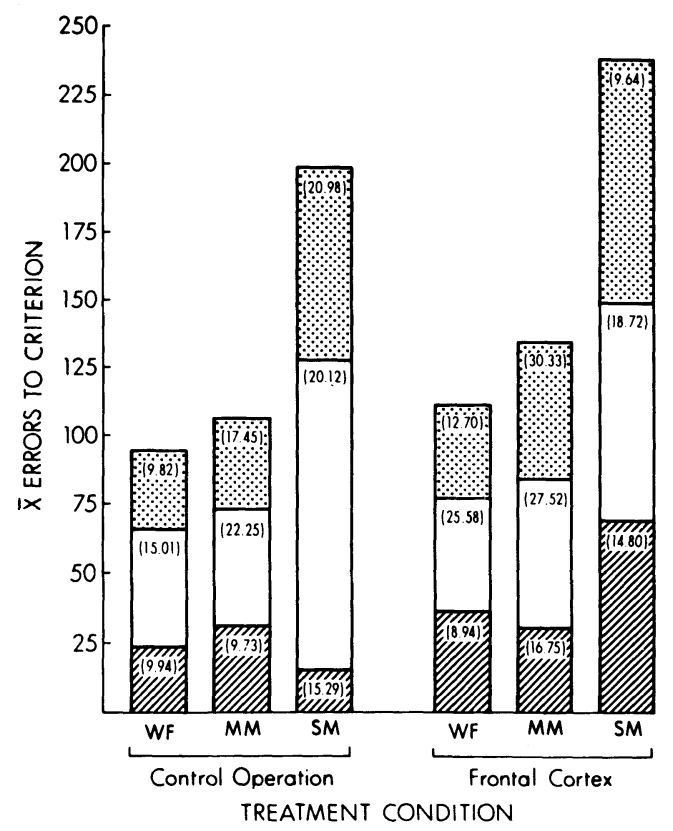

2. Figure 2. Performance scores (mean number of errors to criterion) of the six groups of rats on three reversals of the tactile discrimination. The numbers in brackets are the standard deviations. (First reversal, cross-hatched; second reversal, open; third reversal, stippled.)

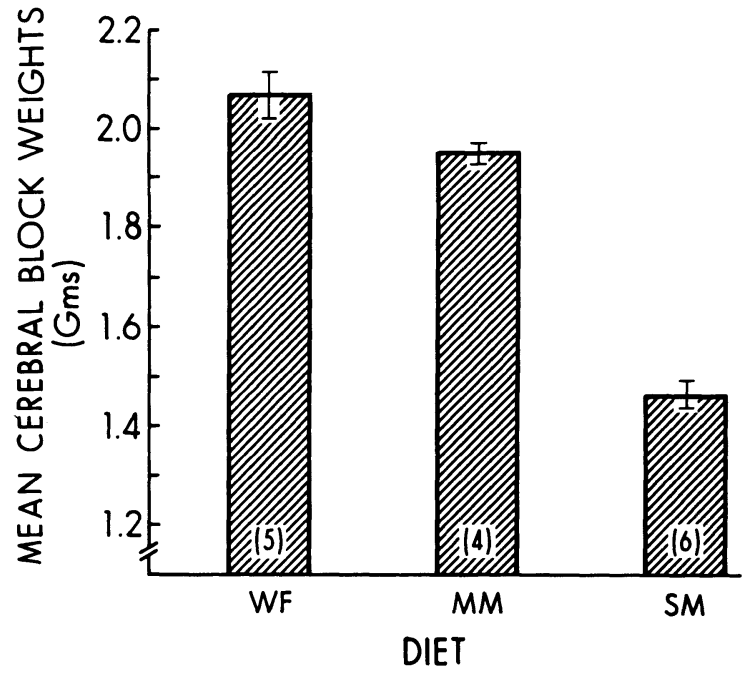

Figure 3. Mean weights of the block of tissue containing the cerebrum and upper brainstem for the WF, MM, and SM groups (200 days of age) with sham operations. Vertical lines on top of bars are standard deviations. Sample sizes are in brackets at bottom.

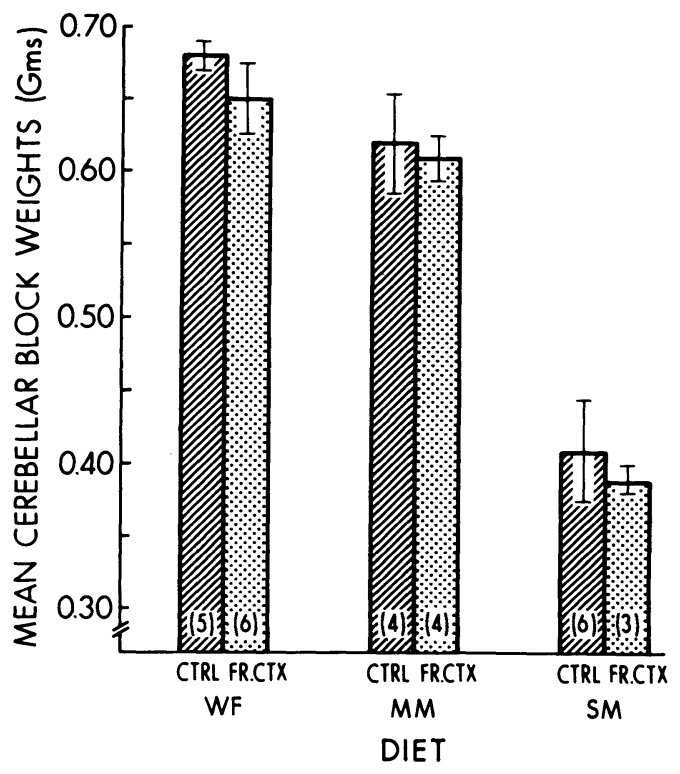

Figure 4. Mean weights of the block of tissue containing the cerebellum and part of the lower brainstem for the WF, MM, and SM groups (200 days of age) with and without lesions of the frontal cortex (control, CTRL; frontal cortex lesion, FR.CTX). Vertical lines on top of bars are standard deviations. Sample sizes are in brackets at bottom.

face were pooled with those first rewarded on the smooth surface. This pooling was based on no differences related to these factors.

The data for original learning are presented in Figure 1. As can be seen, the SM rats with sham operations learned more slowly (i.e., made more errors) than the MM rats with sham operations, although the means of the same two 
Table 1

Means and Standard Deviations of Cerebral Lengths and Widths (in centimeters) of Control-Operated Rats of the Well-Fed (WF), Moderately Malnourished (MM), and Severely Malnourished (SM) Dietary Conditions (200 Days of Age)

\begin{tabular}{lrrr}
\hline & \multicolumn{3}{c}{ Protein Diet Condition } \\
\cline { 2 - 4 } WF & MM & SM \\
\hline Mean & 1.35 & 1.36 & 1.19 \\
SD & .06 & .00 & .03 \\
N & 5 & 4 & 6 \\
& \multicolumn{4}{c}{ Cerebral Length } \\
Mean & 1.39 & 1.37 & \\
SD & .05 & .03 & .03 \\
N & 5 & 4 & 6 \\
\hline
\end{tabular}

groups with frontal cortical lesions were equally poor, with error scores about twice those shown by WF rats. Figure 1 also shows that the sham-operated groups learned in fewer mean trials than the lesion group in each of the three nutritional conditions (WF, MM, SM groups). A $2 \times 3$ ANOVA revealed a significant lesion effect $[F(1,36)=7.90, p<.01]$ and a significant dietary effect $[F(2,36)=9.70, p<.01]$ but no interaction between these factors $[F(2,36)=0.51, p>.05]$. To determine the source of the significant diet effect, Tukey tests were conducted on the scores after surgical conditions had been pooled (based on no significant diet $\times$ lesion interaction), and the results showed that the SM group differed from the WF group $(\mathrm{p}<.01)$ but not from the MM group $(p>.05)$.

The scores of the various groups on the series of three reversals are presented in Figure 2. This figure shows that many more trials were required to reach criterion by the SM groups than by the MM and WF groups, whose scores more closely resembled each other. A $2 \times 3 \times 3$ ANOVA on the reversal data revealed a significant lesion effect $[F(1,33)=4.26, p<.05]$ and a significant nutritional effect $[F(2,33)=27.43, p<.01]$, as well as a task effect $[F(2,66)=7.19, p<.01]$. None of the interactions was statistically significant (all ps $>.05$ ). Tukey tests on the pooled lesion + sham group scores showed that the SM rats performed worse than both the MM and WF groups (ps <.01), and that the MM and WF groups did not differ from each other $(p>.05)$. The basis of the task effect was in the more rapid learning on Reversal 1 (41.4 errors to criterion) than on Reversal $2(54.8)$ or Reversal 3 (51.2).

\section{Brain Measures}

Since there were few female animals in each dietary condition, only the male brains were compared statistically. In addition, brains with cortical lesions were excluded from the analyses involving measurements of cerebral block weight and cerebral width and length.

Figure 3 shows the weights for the block of tissue that included the cerebrum and upper brainstem, and Figure 4 shows the weights for the block that included cerebellum and lower brainstem. Both the cerebral and cerebellar blocks from the SM animals weighed less than those of the MM animals. The ANOVA on cerebral block weight was highly significant $[F(2,12)=116.85, p<.01]$, and Tukey tests showed that the SM group differed from both MM and WF groups at the .01 level. The WF and MM groups did not differ significantly for cerebral block weights $(p>.05)$. The ANOVA on the cerebellar blocks (Figure 4) showed that there was a highly significant main effect of diet $[F(2,22)=62.70, p<.01]$, but no lesion effect $[F(1,22)=1.19, p>.05]$ and no interaction between lesion and early dietary conditions $[\mathrm{F}(2,22)=0.04$, $\mathrm{p}>$.05]. Tukey tests showed that the pooled SM group (lesion + control) differed from the pooled MM and pooled WF groups at the .01 level. The difference between the MM and WF groups was statistically significant for cerebellar block weights $(p<.01)$.

Table 1 summarizes measurements on maximum cerebral length and width for rats in the WF, MM, and SM diet groups. One-way ANOVAS on these dependent variables resulted in highly significant $F$ values $[F s(2,12)=$ 35.23 and 53.62, respectively; ps $<.01]$. Tukey tests showed that the SM group differed from the other two dietary groups on each measure (ps $<.01)$, whereas the MM and WF groups did not differ from each other (ps $>.05)$.

\section{Lesions}

The brains of representative SM rats with frontal cortical lesions are presented in Figure 5. Although brain size and weight were significantly reduced in the SM rats relative to MM and WF rats, the topography and relative size of the frontal cortical lesions were comparable between groups. For each animal, the frontal association cortex was extensively damaged and the lesions did not extend to the motor cortex or below the rhinal fissure. For a few animals in each dietary group, small tissue fragments near the dorsal midline (under and near the midsagittal sinus)

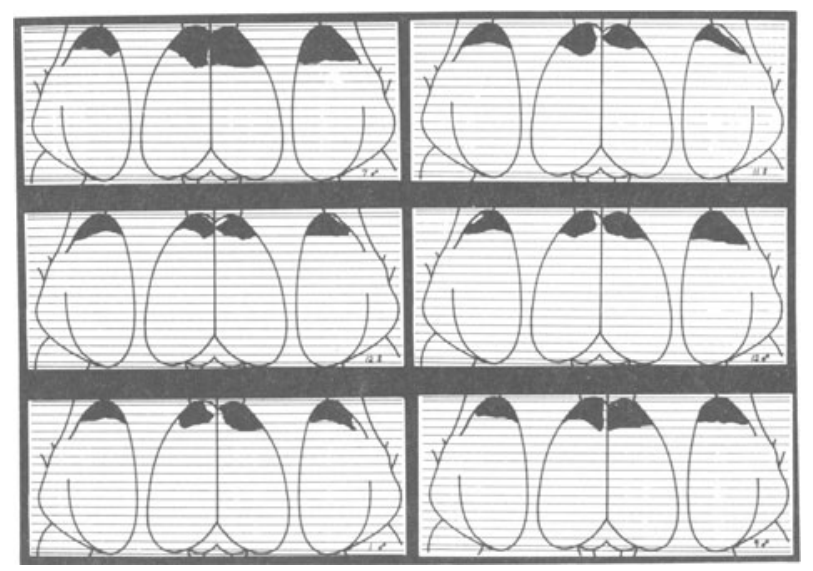

Figure 5. Drawings of the frontal cortical lesions of the rats with lesions in the SM group. (For MM and WF rats, see Silva et al., 1984.) 
or above the rhinal fissure escaped destruction. Subcortically, the lesions infringed upon the forceps major, genu of the corpus callosum, and anterior border of the caudateputamen complex in a few animals of each group. The olfactory bulbs were spared in all animals. No significant correlation was found for lesion size and performance scores. Special attention was paid to the dorsomedial and lateral subfields of the frontal cortex, but here too lesion characteristics that might account for behavioral differences between and within groups were not apparent.

\section{DISCUSSION}

In the present study, there was a main effect of the acute frontal cortex lesion and a main effect of undernutrition on both the original learning and reversal learning tasks. In addition, the rats in the severely malnourished (SM) condition performed more poorly than the moderately malnourished (MM) rats on the reversal series, and the SM rats with frontal cortical lesions were the most impaired of all groups on the reversal series. Measurements of brain size and weights revealed significant differences between the SM and MM rats.

For the well-fed (WF) rats, there was a significant frontal cortical lesion effect for both the acquisition and the reversals of the tactile discrimination. These results complement and extend the results of other experiments which have shown that frontal cortical lesions can disrupt learning with visual and auditory discriminanda (Kling, 1974, 1976), and that the poor reversal learning scores found after these ablations are not restricted to problems with a spatial component (Bourke, 1954; Glick, 1969; Hannon \& Bader, 1974; Jeeves, 1967; Landsell, 1953).

Early malnutrition impaired performance on both the original learning and reversal learning tasks, but the SM animals, which performed worse than the WF rats in both cases, made significantly more errors than the MM animals only on the reversal series. In neither original learning nor reversal learning was there a significant interaction effect involving malnutrition and the frontal cortical lesion, although in both instances the worst scores were those of the animals that had experienced both malnutrition and the focal lesion. Specifically, the SM and MM rats with lesions had the poorest scores in original learning, whereas the SM rats with frontal lesions exhibited the worst scores on the reversal series. Thus, the results of the present study are consistent with those of Mangold et al. (1981) in showing that a history of undernutrition can impair recovery from cortical damage. Nevertheless, they also differ from those of Mangold et al. in that the effects of the lesion and nutrition appeared to be additive under the conditions of this experiment. Diet and lesion were found to be interactive in the Mangold et al. study, which involved $50 \%$ food reduction to the lactating dams and testing for brightness discrimination after posterior cortical lesions. Other lesion-nutrition-interaction effects have been described for rats tested on DRL operant conditioning tasks after hippocampal lesions (Finger \& Green, 1983), although it should be noted that poor early dietary conditions ( $50 \%$ food reduction to lactating dams) did not affect postoperative behavior on other tasks thought to be sensitive to hippocampal lesions (Laughlin, Finger, \& Bell, 1983).

Brain and body-weight measures showed that the SM rats exhibited markedly impaired growth and development, much as had been found by others (Almli \& Yang, 1983; Kesnick \& Morgane, 1983) even for low-birthweight rats that had been cross-fostered at birth and nursed by well-fed mothers. In the present study, there were significant differences among the WF, MM, and SM groups of male rats for body weight at maturity, with the SM animals weighing the least. Among the females, the SM rats weighed less than the MM and WF animals at the time of surgery, although the latter two groups of rats failed to differ from each other. Differences in cerebral and cerebellar "block" weights were found between the WF, MM, and SM groups, with the greatest weight reduction displayed by the SM rats. The SM animals also differed significantly from the MM and WF groups in maximum cerebral length and width; the MM and WF groups did not differ. These gross anatomical measures, obtained 5-6 months after the animals had been dietarily rehabilitated (switched to standard laboratory food), support the contention that some effects of severe undernutrition during the period of rapid brain growth may be permanent, or at least extremely persistent and difficult to overcome.

In summary, the results of the present study showed that the response to an acute frontal cortical lesion sustained at maturity and the effect of early dietary history may be additive under certain circumstances. Animals of the three dietary groups displayed learning deficits in response to frontal cortical lesions sustained later in life. However, the well-fed rats (with lesions) and the undernourished groups (with lesions) did not perform equivalently. That is, the lesion effect was superimposed on an elevated baseline for the malnourished groups. Although it is unfortunate that procedural differences precluded a direct comparison of the $6 \%$ and $8 \%$ casein diet conditions per se, the basic purpose of the present experiment was only to examine a more severely malnourished animal than had been tested earlier (Silva et al., 1984). It is within this context that this study provides new information about the anatomical and behavioral effects of severe malnutrition early in life, and about how early dietary history can form the backdrop against which it is necessary to evaluate the response to a later brain injury. These results stress the importance of knowing more about the condition of the remaining brain when attempting to draw conclusions about the effects of acute brain lesions per se. 


\section{REFERENCES}

Almli, C. R., Henault, M. A., Velozo, C. A., \& Morgane, P. J. (1984). Ontogeny of electrical activity of main olfactory bulb in freelymoving normal and malnourished rats. Developmental Brain Research, in press.

Almli, C. R., \& Montoya, T. (1982). A rat model of small-forgestation-age infants. Paper presented at the meeting of the International Society for Developmental Psychobiology, Minneapolis, MN.

ALMLI, C. R., \& YANG, M. I. (1983). Analysis of sensorimotor behaviors in a rat model of small-for-gestation-age infants. Paper presented at the meeting of the International Society for Developmental Psychobiology, Hyannis, MA.

BoURKE, W. T. (1954). The effects of frontal lobe damage upon habit reversal in the white rat. Journal of Comparative and Physiological Psychology, 47, 227-282.

Davidson, A. N., \& DobBing, J. (1966). Myelination as a vulnerable period in brain development. British Medical Bulletin, 22, 40-44.

Eichenwald, H. F., \& FRY, P. C. (1969). Nutrition and learning. Science, 163, 644-648.

Finger, S., \& Frommer, G. P. (1968). Effects of cortical lesions on tactile discriminations graded in difficulty. Life Science, 7, 897-904.

FINGER, S., \& GREEN, L. (1983). Early undernutrition and later hippocampal damage. I. DRL performance in rats. Nutrition and Behavior, 1, 195-206.

Finger, S., \& STEIN, D. G. (1982). Brain damage and recovery. New York: Academic Press.

GLICK, S. D. (1969). Discrimination learning and reversal in frontal rats as a function of cue. Physiology \& Behavior, 4, 389-392.

HANNON, R., \& BADER, A. (1974). A comparison of frontal pole, anterior median and caudate nucleus in the rat. Physiology \& Behavior, 13, 513-521.

JEEVES, M. A. (1967). Some paradoxical effects of bilateral lesions in the frontal cortex in rats. Neuropsychologia, 5, 73-84..

KLING, J. O. (1974). Effects of frontal ablations on auditory discrimination in the rat. Journal of General Psychology, 91, 75-79.

KLING, J. O. (1976). Effects of frontal ablations upon visual pattern discrimination. Journal of General Psychology, 94, 303-304.

LANSDELL, H. C. (1953). Effects of brain damage on intelligence in rats. Journal of Comparative and Physiological Psychology, 46, 461-464.

Laughlin, N. K., Finger, S., \& Bell, J. (1983). Early undernutrition and later hippocampal damage: Effects on spontaneous behavior and reversal learning. Physiological Psychology, 11, 269-277.

Lundberg, P., Almli, C. R., Morgane, P., Motamedi, M., CatTEGNO, D. (1983). Dietary rehabilitation of malnourished rats: Effects on brain lipid concentrations. Neuroscience Abstracts, 9, 520. Mangold, R., Bell, J., Gruenthal, M., \& Finger, S. (1981). Undernutrition and recovery from brain damage: A preliminary investigation. Brain Research, 230, 406-411.

Miller, M., \& Resnick, O. (1980). Tryptophan availability: The importance of prepartum and postpartum dietary protein on brain indoleamine metabolism in rats. Experimental Neurology, 67, 298-314.

Resnick, O., Miller, M., Forbes, W., Hall, R., Kemper, T., BronZINO, J., \& MoRGANE, P. J. (1979). Developmental protein malnutrition: Influences on the central nervous system of the rat. Neuroscience and Biobehavioral Reviews, 3, 233-246.

ResNick, O., \& MoRgANe, P. (1983). Animal models for small-forgestational-age (SGA) neonates and infants-at-risk (IAR). Developmental Brain Research, 10, 221-225.

Resnick, O., Morgane, P., Hasson, R., Miller, M. (1982). Overt and hidden forms of chronic malnutrition in the rat and their relevance to man. Neuroscience and Biobehavioral Reviews, 6, 55-75.

Silva, M., Bouzrara, A., Finger, S., \& Almli, C. R. (1984). Effects of early protein undernutrition and later frontal cortex damage on habit acquisition and reversal learning in the rat. Physiological Psychology, 12, 141-146.

Stern, W. C., Resnick, O., \& Morgane, P. J. (1976). Developmental protein malnutrition in rats: Acquisition and long-term retention of a single alternation discrimination. Nutrition Reports International, 14, 475-483.

WeST, C., \& KEMPER, T. (1976). The effect of a low protein diet on the anatomical development of the rat brain. Brain Research, 107, 221-237.

WiNICK, M. (1976). Malnutrition and brain development. New York: Oxford University Press.

(Manuscript received September 15, 1984; accepted for publication, pending revision, November 3, 1984.) 